\title{
Comprehension and construct validity of the Visual Prostate Symptom Score (VPSS) by men with obstructive lower urinary tract symptoms in Rural Africa
}

\author{
Lynn Stothers, $\mathrm{MD}^{1}$; S. Mutabazi, $\mathrm{MD}^{2}$; F. Bajunirwe, $\mathrm{MD}^{3}$; Andrew Macnab, MD ${ }^{4}$ \\ ${ }^{1}$ Department of Urologic Sciences and Associate Member School of Population and Public Health, \\ University of British Columbia, Vancouver, BC, Canada, Principal Investigator International Collaboration \\ on Repair Discoveries (ICORD); ${ }^{2}$ Director, Cure Medical Centre, Busheyni, Uganda; ${ }^{3}$ Department of \\ Community Medicine, Mbarara University of Science and Technology, Mbarara, Uganda; ${ }^{4}$ Stellenbosch \\ Institute for Advanced Study, Wallenberg Search Centre, Marais Street, Stellenbosch, South Africa and \\ Associate Member Department of Urologic Sciences, University of British Columbia, Vancouver, BC, \\ Canada
}

Grant Funding: Grand Challenges Canada

Cite as: Can Urol Assoc J 2017; Epub ahead of print. http://dx.doi.org/10.5489/cuaj.4589

Published online November 1, 2017

$* * *$

\section{Abstract}

Introduction: The Visual Prostate Symptom Score (VPSS) is an image-based interpretation of the International Prostate Symptom Score (IPSS) intended to quantify frequency, nocturia, weak stream, and quality of life (QoL) in a literacy-independent manner.

Methods: Ugandan men presenting with lower urinary tract symptoms (LUTS) to a rural clinic completed VPSS and IPSS independently and then with assistance. They verbally interpreted VPSS images, rated question usefulness, and suggested improvements. Responses between word-based and image-based measures were compared (Student's T, Fisher's exact, and Spearman's correlation tests).

Results: 132 scores from 33 men (mean age: 61 years, range 28-93; education: no schooling 20\%, grades 1-4 62\%, 5-7 9\%, 8-12 9\%). Correlation between IPSS and VPSS scores was positive ( $r=0.70)$, as it was between the individual irritative, obstructive, and QoL questions. Independent of education, the weak stream image was best recognized. Likert scale measures indicated this was the most useful image, followed by daytime frequency. Nocturia and QoL images were rated as less clear, with explanation required before most understood that QoL facial expression images reflected overall LUTS impact. Improvements suggested included: increased image size for frequency and nocturia pictograms, increased black/white contrast for nocturia, and addition of an image to allow reporting of urgency. 
Conclusions: In a population with little formal education, there was positive correlation between IPSS and VPSS, with inherent recognition best for weak stream and worst for QoL images. Increased image clarity and an additional image for urgency will enhance the global utility of the VPSS for men to report symptoms of LUTS.

\section{Introduction}

Benign prostatic hypertrophy $(\mathrm{BPH})$ is a widespread urological condition of aging males. ${ }^{1}$ BPH leads to obstruction of voiding and progressive lower urinary tract symptoms (LUTS), which are significantly associated with decreased quality of life (QoL). The International Prostatic Symptom Score (IPSS) was developed by the American Urological Association and later recommended by WHO as the international tool of choice for LUTS assessment. ${ }^{1-3}$ The IPSS is a written score designed to be completed independently by the patient, which comprises 8 questions and quantifies the severity of LUTS, and has been translated into several languages and validated in many populations globally. ${ }^{4-7}$

However, literacy and language fluency are prerequisites to provide accurate and meaningful symptom score responses using the IPSS, Research has established that a minimal education threshold is required for IPSS completion, equivalent to grade 8 education, and that there is an inverse relationship between education level and symptom

question misinterpretation. ${ }^{8,9}$ Problematically the majority of the world's population does not meet this minimal educational threshold. Also, the literature reports that increased misinterpretation occurs when the IPSS is not presented in the patient's primary language. ${ }^{10}$ Even when the IPSS is translated from English into a local language, its uniform validity will often be impacted by the fact that multiple languages are spoken in one region, so even when the translation uses the dominant language, an individual patient's comprehension can still be compromised. Hence, there is an increasing need for a universally accepted way for men to accurately report the presence and severity of their LUTS that is independent of educational achievement and English literacy, especially now, as advanced urologic care entities are increasingly being offered worldwide

The image-based Visual Prostate Symptom Score (VPSS) was developed in 2011 by van de Walt et al. as a literacy-independent adaptation of the IPSS. ${ }^{11}$ The VPSS images correspond to four questions in the IPSS which reflect frequency, nocturia, weak stream and overall quality of life with LUTS. The VPSS has been validated among several cultural groups, been found to be meaningfully correlated with the IPSS, and is more likely to be completed independently by men with lower education. Hence it is a promising tool for men to use who have difficulty completing the IPSS. ${ }^{6}$

The current study aimed to develop VPSS construct and content validity by: evaluating what patients understood each image to represent and comparing these VPSS image descriptions to their IPSS responses. And obtain patient input on how the VPSS 
could be improved to enhance information capture, and quantify impressions of the usefulness of an image-based score in rural Uganda.

\section{Methods}

Ethics was obtained from the Mbarara University of Science and Technology, Uganda. The IPSS was translated into Runyancole, the dominant local language. Participants were men presenting for evaluation of LUTS to the Cure Medical Centre in Busheyni, Sheema county of Uganda. After providing consent they documented their symptoms using the VPSS followed by the IPSS, doing so independently initially and then with the assistance of a member of the clinic staff, and completed a series of evaluative tasks explained to them before they began.

The VPSS image sequences allow the following elements of LUTS to be scored: Q1, frequency; Q2, nocturia; Q3, weak stream; Q4, QoL. These correspond to IPSS questions Q2, Q7, Q5 and Q8 respectively.

The men described verbally to a clinic staff member what they understood the image sequences in each of the VPSS questions to represent, rated the usefulness of this visual representation as a means of reporting their symptoms on a visual analogue scale, and provided feedback on how their comprehension of each image and the overall usefulness of the VPSS could be enhanced. The men also assigned a numeric value to each of the VPSS questions when completing the score independently and again after receiving an explanation of the intended meaning of the images depicted when completing the VPSS with assistance.

Demographic data was collected including: age, level of education and occupation, and body metrics.

Scores were entered into a central database along with the verbal feedback provided by each participant which was content coded. Statistical analysis applied included Student's T, 1-way analysis of variance, the Fisher's exact and the Spearman's correlation tests. The Fisher's exact test analyzed the relationship between education level and variation in total IPSS and VPSS. The Spearman's test determined the degree of correlation between the VPSS and IPSS scores. A p-value of less than 0.05 was set as the threshold for significance.

\section{Results}

Thirty three men native to Sheema district completed the VPSS followed by the IPSS, first independently and then with the assistance of a member of the clinic staff. The demographics of the population and body metrics are shown in Figure 1. Mean age was 61 years (range 28-93); 62\% had a grade 1 to 4 primary education, 9\% grade 8 to 12, 9\% grade 5 to 7 , and 20\% had no formal education. There was a significant difference between the average ages in each educational group as calculated with 1-way analysis of variance test; the men with no education were significantly older than the men who had had primary and secondary education. 
There were no significant differences in IPSS score versus VPSS in the secondary education ( $p=0.83)$, primary education $(0.37)$, nor no education groups $(p=0.35)$.

Spearman's test was applied to various elements of the IPSS and VPSS to determine correlation. It was found that there was a positive correlation between the two symptom scores, most strongly for the irritative voiding questions and the QoL question (Table 2). All four questions from the VPSS had a significant difference in patient's expression of usefulness pre and post-explanation. Men found that the VPSS Q3 image depicting variations in urinary stream was the easiest to comprehend and most useful for reporting their symptoms as it had the lowest difference between pre and postexplanation values (Table 3).

Content coding of feedback on the VPSS showed that the main concern (expressed by 12/33 men - 36\%) was that the VPSS Q2 image sequence for nocturia was difficult to interpret, predominantly due to the depiction of urinary stream being lost in the black background and/or the image being too small to see clearly. The second most reported issue (10/33 men - 30\%) was that a problem of importance to them that they were experiencing was not represented on the VPSS, and hence they could not report it. Most often they were referring to symptoms of urgency. Other comments relevant to utilization of the VPSS included general difficulties with vision within the group which made it difficult to see the detail of the images, especially Q1 frequency and Q2 nocturia. And two subjects did not see the relevance of the QoL facial images as to them they looked like children's faces.

\section{Discussion}

This study shows that in a rural Ugandan community the VPSS is a useful means of capturing some elements of LUTS in men who have limited education and/or literacy. Scores for questions related to urinary stream, and frequency and nocturia correlate between the VPSS and a locally translated version of the IPSS. However, all the IPSS questions are not represented within the VPSS; and an image for urgency was identified to be a relevant addition in particular. Also, the size and clarity of the VPSS images depicting frequency and nocturia limited comprehension. As some degree of visual difficulty is probable in the population for whom VPSS/IPSS assessment is relevant, ${ }^{13}$ making the images larger and clearer with better contrast would increase the usefulness of this visual scale.

Currently, the IPSS is the tool recommended for assessment of LUTS symptoms in men with BPH. However there are clear limitations to its use in populations with limited education and/or literacy, ${ }^{9}$ making the VPSS a valuable alternative in developing countries, where such issues are common.

Ideally, to ensure maximal symptom capture the IPSS would be used after translation into the primary language of the population. However, in Uganda for example there are 31 regional languages spoken. ${ }^{12}$ Also, translating the IPSS requires independent translation and validation steps for each language, and comprehension of the local 
terminology to ensure consistency. In Uganda the population calls the act of urination 'short-call'; without such specific understanding local idiom a translation may be linguistically correct but still fail to convey correct meaning.

Immigrant populations such as those in Canada also need consideration in the context of language-dependent scores. Each major center provides services for many cultural groups. Here too the VPSS would offer a universal method for comprehensive symptom recording and contribute to better urological care.

Feedback in this study provided insight into areas of where confusion over the meaning of images may occur or lack of image clarity could limit their value for symptom reporting. Valid suggestions for improving the VPSS were also forthcoming. Knowing larger or clearer images would help is constructive. Difficulties related to size of print have been found to impact the IPSS. ${ }^{10}$

In developing countries ophthalmologic health services are limited and the population often struggles to maintain their visual acuity and good eye care; ${ }^{13}$ in the Ugandan district studied $>8 \%$ of the population fail Snellen acuity testing and significantly more do not have access appropriate glasses. However, the high comprehension and perceived usefulness of the VPSS Q3 image depicting degrees of impairment of urinary stream confirms the importance of having clear, meaningful images of appropriate size. Hence work to further develop the VPSS appears warranted and this would likely improve its comprehension, increase its value and expand its potential for global use.

Limitations of this study include inclusion of men from only one Ugandan district in Africa. However, the range in the ages and educational attainment of the group recruited would support external generalizability since many similar demographic and social factors are shared with populations in other developing countries.

Importantly, the VPSS provides a validated alternative for evaluation of four elements of the 8 components of LUTS evaluated via the IPSS, and these have inherent value as they allow symptom capture that is independent of any level of educational attainment or specific language literacy. Future efforts to further improve the universality of the VPSS should focus on increasing the number of IPSS elements addressed through images and the clarity of the images. Work is also required to develop a female specific visual symptom score that covers common urinary symptoms experienced by women.

\section{Conclusion}

Construct validity related to immediate recognition is greatest for the slow stream pictogram. Comprehension and reporting would benefit from the addition of an urgency image, increased image size and contrast detail for frequency and nocturia, and a diagrammatic link between the QoL scale to these other constructs. Further development of the VPSS will add to evaluation of LUTS in men in developing countries and amongst immigrant populations, and contribute to the current initiatives promoting the provision of advanced urologic care globally. 


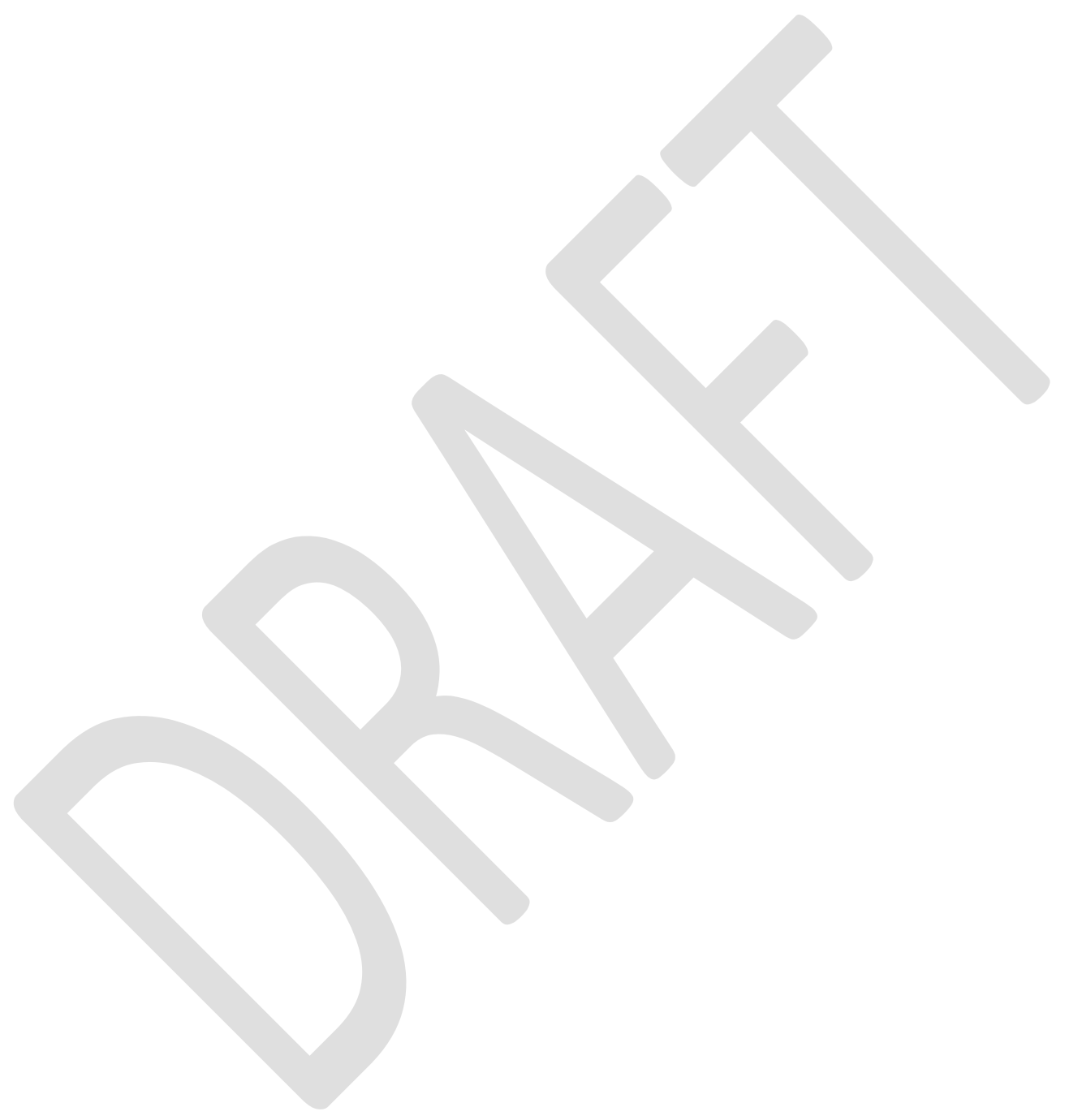




\section{References}

1. Barry, M. J. et al. The American Urological Association symptom index for benign prostatic hyperplasia. The Measurement Committee of the American Urological Association. J. Urol. 148, 1549-57; discussion 1564 (1992).

2. Barry, M. J. et al. Benign Prostatic Hyperplasia Specific Health Status Measures in Clinical Research: How Much Change in the American Urological Association Symptom Index and the Benign Prostatic Hyperplasia Impact Index is Perceptible to Patients? J. Urol. 154, 1770-1774 (1995).

3. Lukacs, B., Grange, J. . \& Comet, D. One-year follow-up of 2829 patients with moderate to severe lower urinary tract symptoms treated with alfuzosin in general practice according to IPSS and a health-related quality-of-life questionnaire. Urology 55, 540-546 (2000).

4. Hammad, F. T. \& Kaya, M. A. Development and validation of an Arabic version of the International Prostate Symptom Score. BJU Int. 105, 1434-1438 (2009).

5. Ozturk, M. I. et al. International Prostate Symptom Score: Really Appreciated by All Patients or...: EBSCOhost. Urol. J. 8, 227-230 (2011).

6. Ceylan, Y. et al. Is New Visual Prostate Symptom Score Useful as International Prostate Symptom Score in the Evaluation of Men With Lower Urinary Tract Symptoms? A Prospective Comparison of 2 Symptom Scores in Turkish Society. Urology 85, 653-658 (2015).

7. Afriansyah, A., Gani, Y. I. \& Nusali, H. Comparison between visual prostate symptom score and international prostate symptom score in males older than 40 years in rural Indonesia. Prostate Int. 2, 176-81 (2014).

8. MacDIARMID, S. A., GOODSON, T. C., HOLMES, T. M., MARTIN, P. R. \& DOYLE, R. B. AN ASSESSMENT OF THE COMPREHENSION OF THE AMERICAN UROLOGICAL ASSOCIATION SYMPTOM INDEX. J. Urol. 159, 873-874 (1998).

9. Netto, N. R. \& de Lima, M. L. The Influence of Patient Education Level on the International Prostatic Symptom Score. J. Urol. 154, 97-99 (1995).

10. Heyns, C. F. et al. Evaluation of the visual prostate symptom score in a male population with great language diversity and limited education: A study from Namibia. South African Med. J. 104, 353 (2014).

11. van der Walt, C. L. E., Heyns, C. F., Groeneveld, A. E., Edlin, R. S. \& van Vuuren, S. P. J. Prospective Comparison of a New Visual Prostate Symptom Score Versus the International Prostate Symptom Score in Men With Lower Urinary Tract Symptoms. Urology 78, 17-20 (2011).

12. Ladefoged, P. Another view of endangered languages. Source Lang. 68103160, 809-811 (1992).

13. Mbulaiteye, S. M., Reeves, B. C., Mulwanyi, F., Whitworth, J. A. G. \& Johnson, G. Incidence of visual loss in rural southwest Uganda. Br. J. Ophthalmol. 87, 82933 (2003). 


\section{Figures and Tables}

Fig. 1. Visual Prostate Symptom Score (VPSS) consisting of Q1: frequency, Q2: nocturia, Q3: stream and Q4: quality of life. Q1: frequency and Q2: nocturia represent irritative symptoms and Q3: stream represents the obstructive symptoms.
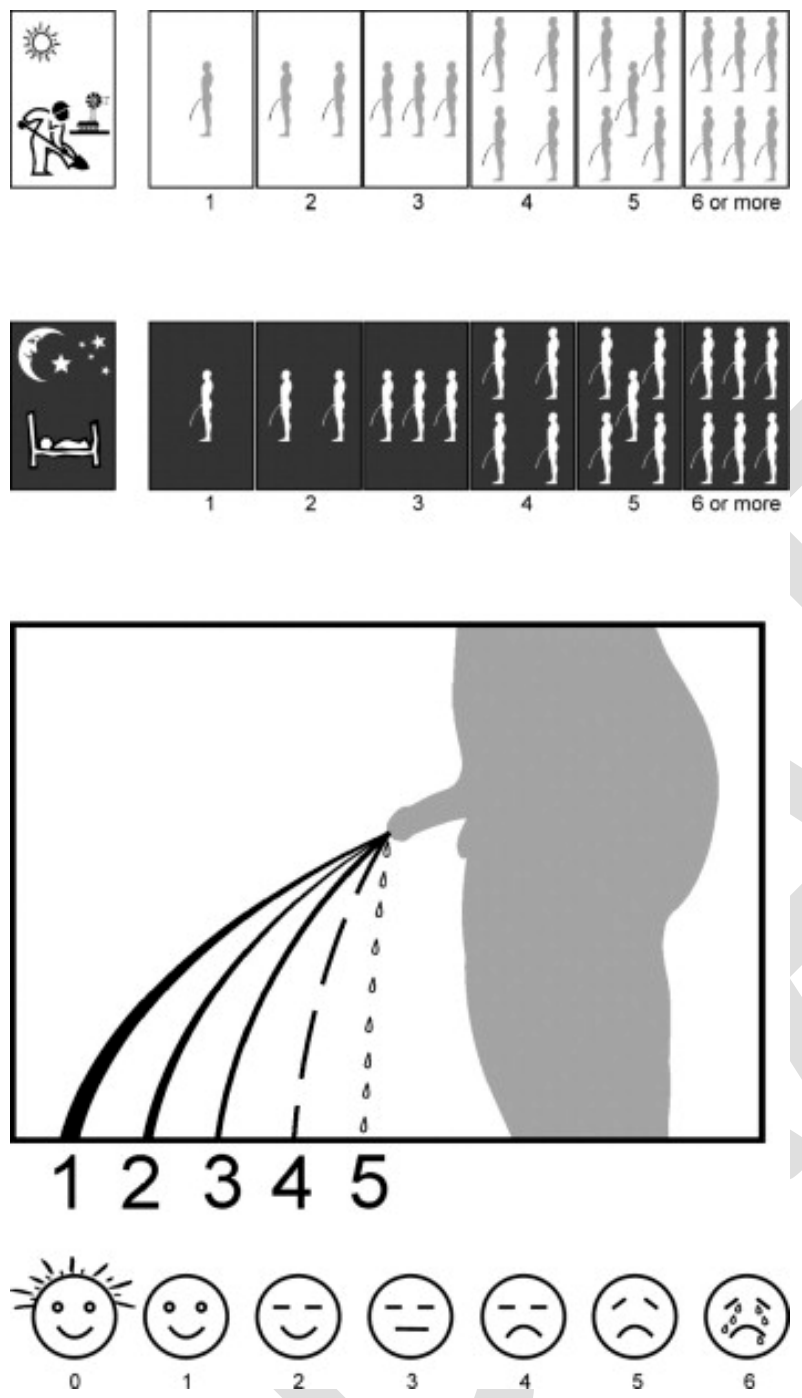
Table 1. Population demographics. Mean age, range, education level, body metrics, and occupation (total $\mathbf{n}=33$ )

\begin{tabular}{|c|c|c|c|c|c|c|}
\hline $\begin{array}{c}\text { Education } \\
\text { level }\end{array}$ & $\mathbf{\%}$ (N) & $\begin{array}{c}\text { Age } \\
\text { (Years) }\end{array}$ & $\begin{array}{c}\text { SD of } \\
\text { Age }\end{array}$ & $\begin{array}{c}\text { Range } \\
\text { (Years) }\end{array}$ & $\begin{array}{c}\text { Mean } \\
\text { BMI }\end{array}$ & Occupations \\
\hline Secondary & $9 \%(3)$ & 61.67 & 29.87 & $29-71$ & 23 & $\begin{array}{c}\text { Mechanical } \\
\text { Engineer N=1 } \\
\text { Business N=1 }\end{array}$ \\
\hline Primary & $\begin{array}{c}61 \% \\
(20)\end{array}$ & 55.95 & 11.23 & $28-85$ & 21 & $\begin{array}{c}\text { Peasant N=16 } \\
\text { Business N=1 } \\
\text { Mechanic } \\
\text { N=1 } \\
\text { Farmer N=2 }\end{array}$ \\
\hline None/Unknown & $\begin{array}{c}30 \% \\
(10)\end{array}$ & 71.8 & 16.64 & $39-93$ & 20 & Peasant N=9 \\
\hline Total & 33 & 61.27 & 16.15 & $28-93$ & 21 & \\
\hline$p \quad$ & & $0.03^{*}$ & & & & \\
\hline
\end{tabular}

*Calculated with a one-way analysis of variance test.

\begin{tabular}{|l|c|}
\hline $\begin{array}{l}\text { Table 2. Total, irritative, obstructive and QoL correlation } \\
\text { between IPSS and VPSS }\end{array}$ \\
\hline Spearman's correlation & Correlation coefficient $(r)$ \\
\hline $\begin{array}{l}\text { Irritative IPSS vs. VPSS } \\
\text { • Q2 IPSS vs. Q1 }\end{array}$ & 0.68 \\
VPSS & 0.81 \\
Q7 IPSS vs Q2 & 0.66 \\
\hline VPSS & 0.76 \\
\hline Obstructive IPSS vs. VPSS & 0.53 \\
- Q1 IPSS vs. VPSS & 0.76 \\
- Q3 IPSS vs. VPSS & 0.73 \\
- Q6 IPSS vs. VPSS & 0.74 \\
\hline QoL IPSS vs. VPSS & 0.70 \\
\hline
\end{tabular}

Calculated using the Spearman's rank correlation test. Irritative symptoms denoted by the IPSS Q2, Q4 and Q7 and the VPSS Q1 \&Q2. Obstructive symptoms IPSS Q1, Q3, Q5 \& Q6 and VPSS Q3. 
Table 3. Difference in perceived VPSS question usefulness pre and post-explanation

\begin{tabular}{|c|c|c|c|c|}
\hline & Q1: Frequency & Q2: Nocturia & Q3: Stream & $\begin{array}{c}\text { Q4: Quality of } \\
\text { Life }\end{array}$ \\
\hline $\begin{array}{c}\text { Average } \\
\text { difference }\end{array}$ & 4.29 & 3.75 & 2.00 & 4.90 \\
\hline $\begin{array}{c}\text { Standard } \\
\text { deviation }\end{array}$ & 2.84 & 2.04 & 2.11 & 2.78 \\
\hline$p$ & $9.74 \mathrm{E}-11^{*}$ & $2.70 \mathrm{E}-13^{*}$ & $3.09 \mathrm{E}-06^{*}$ & $1.09 \mathrm{E}-12^{*}$ \\
\hline
\end{tabular}

Calculated via difference in assigned numerical value and Student's T test. Significance $\mathrm{p}<0.05$. 